DE

M E D I C I N A

T R O P I C A L

$\mathrm{DE}$

SÃO PAULO

JOURNAL OF THE SÃO PAULO INSTITUTE OF TROPICAL MEDICINE

(1) Universidade Federal de São Paulo, Departamento de Pediatria, São Paulo, São Paulo, Brazil

(2) University of Miami, Department of Medicine, Miami, USA

Correspondence to: Maria Isabel de Moraes-Pinto

Universidade Federal de São Paulo, Departamento de Pediatria, Divisão de Infectologia Pediátrica, Rua Pedro de Toledo, 781, 9ำ andar, CEP 04039-032, Vila Clementino, São Paulo, SP, Brazil Tel: +5511 5576-4848 VOIP 3044, Fax: +55 $115575-6928$

E-mail:m.isabelmp@uol.com.br

Received: 18 July 2016

Accepted: 22 February 2017

\section{Immune development in HIV-exposed uninfected children born to HIV-infected women}

\author{
Maristela Miyamoto', Aída F.T.B. Gouvêa', Erika Ono', Regina Célia M. \\ Succi ${ }^{1}$, Savita Pahwa², Maria Isabel de Moraes-Pinto'
}

\section{ABSTRACT}

Immunological and clinical findings suggestive of some immune dysfunction have been reported among HIV-exposed uninfected (HEU) children and adolescents. Whether these defects are persistent or transitory is still unknown. HEU pediatric population at birth, 12 months, 6-12 years were evaluated in comparison to healthy age-matched HIV-unexposed controls. Plasma levels of LPS, sCD14, cytokines, lymphocyte immunophenotyping and T-cell receptor excision circles (TREC) were assessed. HEU and controls had similar LPS levels, which remained low from birth to 6-12 years; for plasma sCD14, IL-2, IL-6, IL-7, IL-10, IL-12p70, IL-13, IL-17, IFN- $\gamma$, TNF- $\alpha$, G-CSF, GM-CSF and MCP-1, which increased from birth to 12 months and then decreased at 6-12 years; and for TREC $/ 10^{6}$ PBMC at birth in HEU and controls. By contrast, plasma MIP-1 $\beta$ levels were lower in HEU than in controls $(\mathrm{p}=0.009)$ at 12 months, and IL-4 levels were higher in HEU than controls $(\mathrm{p}=0.04)$ at 6-12 years. Immune activation was higher in HEU at 12 months and at 6-12 years than controls based on frequencies of CD38+HLA-DR+CD8+T cells $(\mathrm{p}=0.05)$ and of CD38+HLA-DR+CD4+T cells ( $\mathrm{p}=0.006)$. Resting memory and activated mature B cells increased from birth to 6-12 years in both groups. The development of the immune system in vertically HEU individuals is comparable to the general population in most parameters, but subtle or transient differences exist. Their role in influencing clinical incidences in HEU is unknown.

KEYWORDS: HIV-exposed uninfected. Lymphocyte activation. Cytokines. Lipopolysaccharide. CD14 antigen.

\section{INTRODUCTION}

It is now well established that exposure to different pathogens (even in the absence of infection), use of antibiotics (administered both as prophylaxis and as treatment) and different dietary habits might direct the immune system towards different maturation profiles ${ }^{1,2}$. Infants born to women with human immunodeficiency virus (HIV) infection are associated with many such factors, with exposure to maternal HIV infection and associated coinfections ${ }^{3}$, maternal cytokine environment ${ }^{4}$, maternal vaginal microbiome ${ }^{5,6}$, antiretroviral drugs $^{7}$, antibiotics used as prophylaxis ${ }^{8}$ and to administration of formula since birth in many countries. It is still not clear however how the fetus/ neonate is impacted by the degree of exposure to HIV during pregnancy, puerperium and early infancy and what are the immune consequences of this experience ${ }^{9,10}$.

Literature on this topic has been inconclusive. A few HIV-exposed uninfected (HEU) children have been shown to present clinical symptoms and 
diagnosis suggestive of some immune dysfunction such as Pneumocystis jiroveci pneumonia and Pseudomonas aeruginosa sepsis $^{11}$, or a trend to more frequent low respiratory tract infections ${ }^{12}$. Other studies have suggested laboratory alterations, such as reduced $\mathrm{CD} 4+\mathrm{T}^{13}$ and $\mathrm{B}$ cell counts $^{11}$, higher immune activation ${ }^{14,15}$ and higher apoptosis in $\mathrm{B}$ cells ${ }^{13}$. It is unclear whether such alterations are transitory or if they persist beyond infancy. Our findings are informative for hitherto undescribed immune development in these children.

\section{MATERIAL AND METHODS}

\section{Patients and study design}

In this study, we have evaluated the presence of lymphocyte immune activation in HEU children and adolescents by assessing microbial translocation (lipopolysaccharide, LPS levels), monocyte activation (plasma sCD14 levels), plasma cytokine levels, and lymphocyte immunophenotyping in different age strata. We have also evaluated thymic function by quantifying T-cell receptor excision circles (TRECs) in cord blood samples.

We used a repository of frozen plasma and cryopreserved peripheral blood mononuclear cell (PBMC) samples from preview studies in this protocol, which was approved by the Ethics Committee of the Federal University of São Paulo, in São Paulo, Brazil (protocol numbers 851/01 and 1612/04). Written informed consent was obtained from all parents prior to enrollment of children in the previous studies.

Vertically HIV-exposed uninfected individuals were assessed at birth (HEU-Birth = 19), at 12 months of age (HEU-12 months $=19$ ) and at 8.5 years of age (HEU-6-12 years $=20$ ). They were compared to age matched nonHIV-exposed healthy children at birth $($ Control-Birth $=20)$, at 12 months (Control-12 months $=19)$ and at 8.5 years $($ Control-6-12 years $=18)($ Table 1$)$.

All HEU individuals evaluated at birth and at 12 months, and 11 out of 20 (55\%) 6-12 years strata received zidovudine for 6 weeks after birth, as prophylaxis against motherto-child HIV transmission. All HEU children from the three strata received sulfamethoxazole/ trimethoprim (SMZ-TMP) three times a week from then on until six months of age as prophylaxis against $P$. jiroveci motherto-child HIV transmission.

Children of different ages born to healthy HIV negative mothers after uneventful pregnancies without evidence of previous serious disease were enrolled as controls. At assessment, they did not present any infection and were not on any medication.

HEU children were regularly followed-up at the Pediatric AIDS Outpatient Clinic of the Federal University of São Paulo and they were tested for HIV infection using HIV DNA PCR during the first semester of life and/or ELISA kits for HIV antibodies after 18 months of age. They were considered uninfected after two negative tests.

Besides that, at the study entry, all children from the Control and the HEU groups were tested for HIV using ELISA kits to HIV antibodies.

\section{Plasma LPS analysis}

Plasma samples were diluted to $20 \%$ with endotoxin-free water and were then warmed to $70^{\circ} \mathrm{C}$ for $10 \mathrm{~min}$ to inactivate plasma proteins. Plasma samples were tested in duplicate with a commercially available Limulus Amebocyte assay (Cambrex Bioscience, Walkersville, USA) according to the manufacturer's protocol. Measurements were obtained on a kinetic absorbance reader at $450 \mathrm{~nm}$ (ELX800; Bio-Tek Instruments, Vermont, USA).

Table 1 - Demographic and clinical characteristics of control and HEU group children at different age strata

\begin{tabular}{|c|c|c|c|c|c|c|c|}
\hline \multirow[b]{2}{*}{ Parameters } & \multicolumn{2}{|c|}{ Birth } & \multicolumn{2}{|c|}{$12 \mathrm{mo}$} & \multicolumn{2}{|c|}{$6-12 y$} & \multirow{2}{*}{$\begin{array}{c}\text { p value for comparisons } \\
\text { between Control and } \\
\text { HEU groups }\end{array}$} \\
\hline & $\begin{array}{l}\text { Control } \\
(n=20)\end{array}$ & $\begin{array}{c}\text { HEU } \\
(n=19)\end{array}$ & $\begin{array}{l}\text { Control } \\
(n=19)\end{array}$ & $\begin{array}{c}\text { HEU } \\
(n=19)\end{array}$ & $\begin{array}{l}\text { Control } \\
(n=18)\end{array}$ & $\begin{array}{c}\text { HEU } \\
(n=20)\end{array}$ & \\
\hline Median age in years (range) & $\begin{array}{c}0 \\
(0.0-0.0)\end{array}$ & $\begin{array}{c}0 \\
(0.0-0.0)\end{array}$ & $\begin{array}{c}1.0 \\
(0.9-1.0)\end{array}$ & $\begin{array}{c}1.0 \\
(1.0-1.0)\end{array}$ & $\begin{array}{c}8.5 \\
(7.0-10.8) \\
\end{array}$ & $\begin{array}{c}8.5 \\
(7.8-10.0)\end{array}$ & $p>0.05$ \\
\hline Number of females (\%) & $\begin{array}{c}12 / 20 \\
(60) \\
\end{array}$ & $\begin{array}{c}12 / 19 \\
(63) \\
\end{array}$ & $\begin{array}{l}9 / 19 \\
(47)\end{array}$ & $\begin{array}{c}12 / 19 \\
(63) \\
\end{array}$ & $\begin{array}{c}10 / 18 \\
(56)\end{array}$ & $\begin{array}{c}10 / 20 \\
(50)\end{array}$ & $p>0.05$ \\
\hline Maternal smoking (\%) & $\begin{array}{c}0 / 20 \\
(0)\end{array}$ & $\begin{array}{l}7 / 19 \\
(37)\end{array}$ & $\begin{array}{l}3 / 19 \\
(16)\end{array}$ & $\begin{array}{l}7 / 19 \\
(37)\end{array}$ & UNK & UNK & 0.003 (at birth) \\
\hline Cesarean section (\%) & $\begin{array}{c}1 / 20 \\
(5)\end{array}$ & $\begin{array}{l}19 / 19 \\
(100)\end{array}$ & UNK & $\begin{array}{l}19 / 19 \\
(100)\end{array}$ & UNK & $\begin{array}{l}3 / 8 \\
(36)^{*}\end{array}$ & $<0.0001$ (at birth) \\
\hline Breastfeeding $(\%)^{\star *}$ & NA & NA & $\begin{array}{c}17 / 19 \\
(89)\end{array}$ & $\begin{array}{c}0 / 19 \\
(0)\end{array}$ & UNK & $\begin{array}{c}1 / 8 \\
(12)^{\star \star}\end{array}$ & $<0.0001$ (at 12 months) \\
\hline
\end{tabular}

UNK = unknown information. * Unknown information for 12 individuals. ${ }^{* *}$ Individuals who received exclusive or mixed breastfeeding. 


\section{Plasma sCD14 analysis}

Plasma sCD14 levels were determined using human sCD14 ELISA kit (R\&D Systems, Minneapolis, USA), according to the manufacturer's specifications. Samples were run in duplicate on a kinetic absorbance reader at $450 \mathrm{~nm}$ (ELX800; Bio-Tek Instruments, Vermont, USA).

\section{Plasma cytokine levels}

Plasma cytokine (IL-1 $\beta$, IL-2, IL-4, IL-5, IL-6, IL-7, IL-8, IL-10, IL-12 p70, IL-13, IL-17, G-CSF, GM-CSF, IFN- $\gamma$, MCP-1, MIP- $1 \beta$ and TNF- $\alpha$ ) levels were determined using Bio-Plex Pro Human cytokine 17-plex assay (Bio-Rad, Hercules, USA), according to the manufacturer's specifications. Measurements were obtained on a Bio Plex 200 Suspension Array System (Bio-Rad, Austin, USA).

\section{Flow cytometry analyses}

Cryopreserved PBMC were thawed and processed if they had at least $85 \%$ viability. They were assessed by 6-color flow cytometry on the same day (FACSCanto, BD Biosciences, Franklin Lakes, USA) after cell staining and they were analyzed using the FlowJo software (TreeStar, San Jose, USA).

The following monoclonal antibodies were used to assess T, B and NK cells: CD3-FITC, CD4-PerCP, CD10-PE-Cy7, CD19-PerCP, CD38-FITC, CD38-PE-Cy7 (BD Biosciences, San Jose, USA); CD3-APC-Cy7, CD4-FITC, CD16-PE-Cy7, CD21-APC, CD27-PE, CD45-APC-Cy7, CD56-PE-Cy7, HLA-DR-PerCP, PD-1-PE (BD, San Jose, USA); 2B4-APC and FcRL4-APC (BioLegend, San Diego, USA). Isotypic controls (IgG1-FITC, IgG1-PE, IgG2a-PerCP, IgG1-PE-Cy7, IgG1-APC, IgG2b-APC, from BD Biosciences, San Jose, USA; IgG1-PE and IgG1-APC, from BioLegend, San Diego, USA) were used to evaluate nonspecific staining.

CD4+T cells were defined as CD3+CD4+ and CD8+T cells were defined as CD3+CD4-, and evaluated for frequencies of activated (CD38+HLA-DR+); or exhausted (PD-1+ or $2 \mathrm{~B} 4+$ ) cell subset. B cells were defined as CD3-CD19+, and evaluated for frequencies of transitional (CD10+), naïve (CD10-CD21+CD27-), resting memory (CD10-CD21+CD27+), activated mature (CD10-CD21$\mathrm{CD} 27+)$ and exhausted memory B cells (CD10-CD21CD27- or PD-1+ or FcRL4+).

NK cells were defined as CD45+CD3-CD16+CD56+ and evaluated for frequencies of PD-1+ exhausted cells.

\section{Quantification of TRECs}

DNA was extracted from frozen PBMCs using the QIAamp DNA Mini and Blood Mini kit (QIAgen, Valencia, USA). TRECs were quantified by realtime PCR, in an ABI 7500 Real Time PCR system (Applied Biosystems, Foster City, USA). In brief, $0.5 \mathrm{mM}$ primers (CACATCCCTTTCAACCATGCT and GCCAGCTGCAGGGTTTAGG) (Sigma-Aldrich, Saint Louis, USA), $0.2 \mathrm{mM}$ TaqMan probe FAMACACCTCTGGTTTTTGTAAAGGTGCCCACT-BHQ1 (Sigma-Aldrich, Saint Louis, USA), 0.025 U of Platinum Taq polymerase, $3.5 \mathrm{mM} \mathrm{MgCl} 2,0.2 \mathrm{mM}$ dNTPs in TaqMan buffer (Invitrogen, Carlsbad, USA) and $5 \mathrm{uL}$ of genomic DNA were mixed in a total volume of $25 \mathrm{uL}$. A total of 5 $\mathrm{uL}$ of DNA was used for the TREC assay. PCR conditions were $95{ }^{\circ} \mathrm{C}$ for $5 \mathrm{~min}$. and 40 cycles of $95^{\circ} \mathrm{C}$ for $30 \mathrm{sec}$., and $60^{\circ} \mathrm{C}$ for $1 \mathrm{~min}$. A standard curve was included in every $\mathrm{PCR}$ reaction for the absolute quantification of the number of TRECs per $10^{6}$ cells of PBMC in each sample. TREC fragments were generously provided by Dr. Daniel Douek. The TREC standard curve was established using seven 10fold dilutions ranging from $10^{2}$ to $10^{8}$ TREC copies $/ 5 \mathrm{uL}$ of plasmids containing the TREC fragment. Each sample was run in duplicate and average TREC values were used for data analysis. Results were expressed as TRECs per $10^{6}$ cells. During the process of assay standardization, TRECs from cord blood lymphocytes were compared to PBMC samples from healthy adults aged 23 to 35 years.

\section{Statistical analysis}

Continuous variables were analyzed by the MannWhitney test; if more than two groups were tested, KruskalWallis test (KW) and Student-Newman-Keuls were used. Categorical variables were analyzed by the chi-squared test.

Pearson's correlation coefficient was assessed for $\mathrm{CD} 4+\mathrm{T}$ cell counts and frequencies of activated or exhausted subsets.

For all analyses, Minitab 14 (Minitab, State College, USA) and BioEstat 5.0 (Instituto de Desenvolvimento Sustentável Mamirauá, Tefé, Brazil) were employed. The level of significance was set at $\mathrm{p}<0.05$.

\section{RESULTS}

\section{Demographic data}

Demographic and clinical data are presented on Table 1. In the vast majority of mothers (77\%) of two groups of HEU children (birth and 12-month groups), HIV viral 
loads close to delivery were lower than 400 copies $/ \mathrm{mL}$. In the other $23 \%$ of mothers, viral load varied from 1,310 to 26,400 copies/ $\mathrm{mL}$. No information on maternal viral load was available for the 6-12 years old strata.

Seventeen infants (89\%) from control-12 months group had exclusive or mixed breastfeeding for a median of 6.8 months; by contrast, no child from HEU-12 months group was breastfed $\left(\chi^{2}, \mathrm{p}<0.0001\right)$. Breastfeeding information was not available from the 6-12 years control-group. Only one individual from the HEU-6-12 earsy group was breastfed for 3 days, which occurred before his mother was diagnosed with HIV infection (Table 1).

\section{Plasma LPS and SCD14 levels}

Plasma LPS levels were similar among HEU and control children and did not vary with age from birth until 6-12 years of age (KW, p=0.189) (Figure 1). By contrast, plasma sCD14 levels increased significantly from birth to 12 months of age independently of HIV exposure (Figure 1). A slight decay in plasma sCD14 levels was observed from 12 months to 6-12 years, but that was only significant for HEU children (Figure 1). No differences were noted between HEU and C-control groups within any age strata (Figure 1).

\section{Plasma cytokine levels}

Plasma levels from 17 cytokines were assessed ex vivo, without any in vitro stimulus. The majority of cytokines (IL-1 $\beta$, IL-2, IL-5, IL-6, IL-7, IL-8, IL-10, IL-12 p70, IL-13, IL-17, IFN- $\gamma$, TNF- $\alpha$, G-CSF, GM-CSF and MCP-1) showed some variation with age and many of them peaked

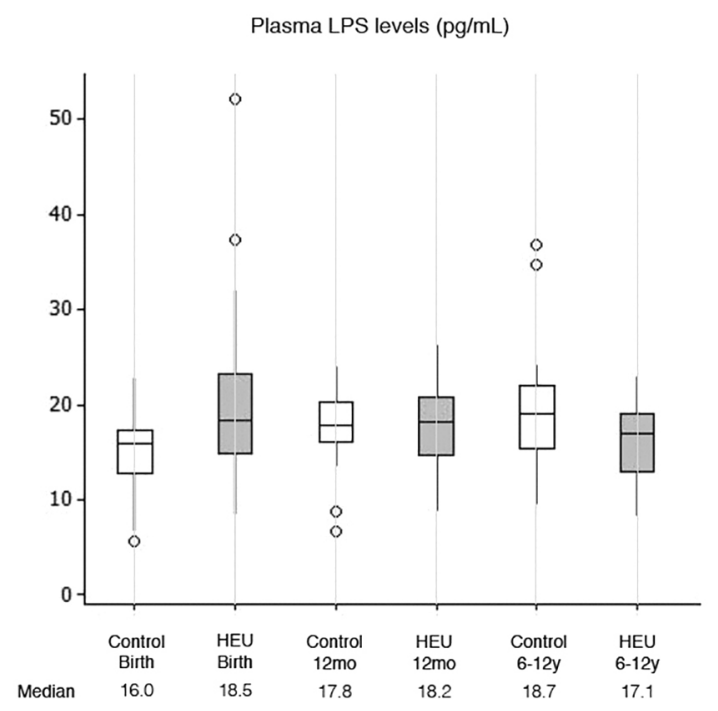

at 12 months of age (IL-2, IL-6, IL-7, IL-10, IL-12 p70, IL-13, IL-17, IFN- $\gamma$, TNF- $\alpha$, G-CSF, GM-CSF and MCP-1) (Table 2). The HEU group differed from the control group with respect to two cytokines on different occasions: median IL-4 levels were higher at 6-12 years in HEU than in C-controls ( 1.0 versus $0.6 \mathrm{pg} / \mathrm{mL} ; \mathrm{KW}, \mathrm{p}=0.043$ ); median MIP- $1 \beta$ levels, by contrast, were lower in HEU at 12 months when compared with C-controls (23.9 versus $46.0 \mathrm{pg} / \mathrm{mL}$; KW, $\mathrm{p}=0.009$ ).

IL-5 was the only cytokine from which no statistical differences were noted among groups at any age (KW, $\mathrm{p}=0.588$ ).

\section{Activation and exhaustion markers by flow cytometry}

An increase in the expression of activation markers was observed on CD8+ T cells from birth to 12 months of age for HEU followed by a decrease at 6-12 years in HEU $(\mathrm{p}=0.011$ and $\mathrm{p}=0.001$, respectively) (Table 3$)$. CD $4+\mathrm{T}$ cell effects were less striking but followed a similar pattern. The HEU group had a higher frequency of CD38+HLA-DR+ $\mathrm{CD} 8+\mathrm{T}$ cells than controls at 12 months of age $(\mathrm{p}=0.053)$ and a higher frequency of CD38+HLA-DR+CD4+T cells than controls at $6-12$ years $(\mathrm{p}=0.006)$.

PD-1 expression on CD4+ T cells increased at 12 months and was maintained on that level until 6-12 years (Table 3). On the other hand, PD-1 expression on CD8+ T cells increased at 12 months and decreased at 6-12 years (Table 3). 2B4 expression on CD4+ T cells increased at 12 months in controls and at 6-12 years in HEU (Table 3); 2B4 expression on CD8+ T cells increased at 12 months and was maintained on that level until 6-12 years (Table 3).

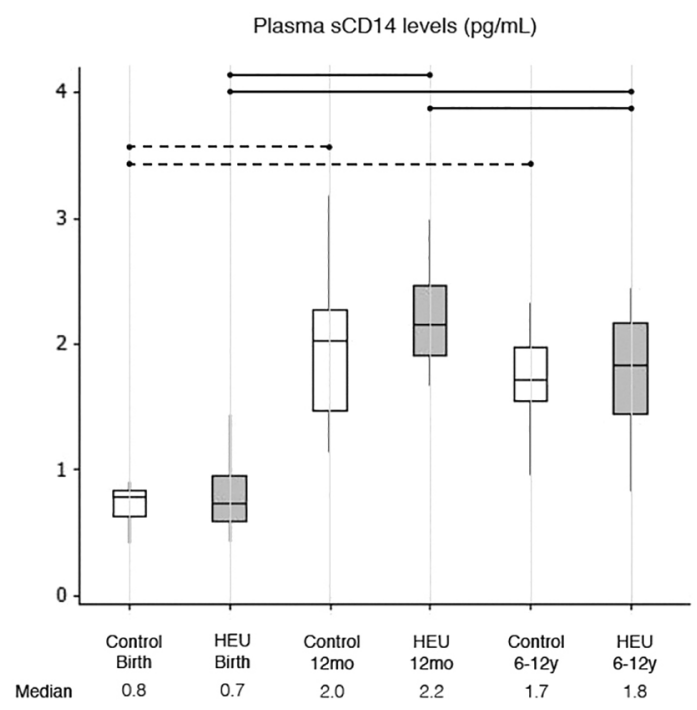

Figure 1 - Plasma LPS levels and SCD14 levels in control and HEU groups at different ages (Birth, 12mo and 6-12y). Outliers are represented by circles. Differences between HEU children at different age strata are shown as solid lines; differences between control children at different age strata are shown as dashed lines 
Table 2 - Median plasma cytokines in control and HEU groups at different ages, with interquartile values in parenthesis

\begin{tabular}{|c|c|c|c|c|c|c|c|c|c|}
\hline & \multicolumn{2}{|c|}{ Birth } & \multicolumn{2}{|c|}{$12 \mathrm{mo}$} & \multicolumn{2}{|c|}{$6-12 y$} & \multicolumn{3}{|c|}{$p$ values } \\
\hline & $\begin{array}{l}\text { Control } \\
(\mathrm{n}=20)\end{array}$ & $\begin{array}{c}\text { HEU } \\
(n=19)\end{array}$ & $\begin{array}{l}\text { Control } \\
(n=19)\end{array}$ & $\begin{array}{c}\text { HEU } \\
(n=19)\end{array}$ & $\begin{array}{l}\text { Control } \\
(n=18)\end{array}$ & $\begin{array}{c}\text { HEU } \\
(n=20)\end{array}$ & $\begin{array}{l}\text { HEU vs. } \\
\text { Control }\end{array}$ & $\begin{array}{c}\text { Control at different } \\
\text { ages }\end{array}$ & $\begin{array}{l}\text { HEU at different } \\
\text { ages }\end{array}$ \\
\hline IL-1 $1 \beta$ & $\begin{array}{c}1.8 \\
(1.0,6.5) \\
\end{array}$ & $\begin{array}{c}1.9 \\
(0.9,6.3) \\
\end{array}$ & $\begin{array}{c}1.0 \\
(0.8,1.4) \\
\end{array}$ & $\begin{array}{c}1.2 \\
(0.7,1.1) \\
\end{array}$ & $\begin{array}{c}0.8 \\
(0.5,1.1) \\
\end{array}$ & $\begin{array}{c}0.9 \\
(0.7,1.2) \\
\end{array}$ & $p>0.05$ & $\begin{array}{l}0.014 \text { (birth-12mo) } \\
0.001 \text { (birth-6-12y) }\end{array}$ & 0.001 (birth-6-12y) \\
\hline IL-2 & $\begin{array}{c}2.0 \\
(1.7,2.1) \\
\end{array}$ & $\begin{array}{c}2.0 \\
(1.5,2.0) \\
\end{array}$ & $\begin{array}{c}2.4 \\
(2.0,4.5) \\
\end{array}$ & $\begin{array}{c}2.9 \\
(2.0,6.4) \\
\end{array}$ & $\begin{array}{c}2.0 \\
(1.5,3.0) \\
\end{array}$ & $\begin{array}{c}2.0 \\
(2.0,3.0) \\
\end{array}$ & $p>0.05$ & 0.038(birth-12mo) & 0.006(birth-12mo) \\
\hline IL-4 & $\begin{array}{c}0.9 \\
(0.6,1.3) \\
\end{array}$ & $\begin{array}{c}0.7 \\
(0.6,1.1) \\
\end{array}$ & $\begin{array}{c}1.0 \\
(0.7,1.2) \\
\end{array}$ & $\begin{array}{c}1.1 \\
(0.8,1.6) \\
\end{array}$ & $\begin{array}{c}0.6 \\
(0.5,0.9) \\
\end{array}$ & $\begin{array}{c}1.0 \\
(0.7,1.1) \\
\end{array}$ & $\begin{array}{c}0.043(6- \\
12 y)\end{array}$ & $\begin{array}{c}0.024 \text { (birth-6-12y) } \\
0.004(12 \mathrm{mo}-6-12 \mathrm{y})\end{array}$ & 0.017 (birth-12mo) \\
\hline IL-5 & $\begin{array}{c}4.3 \\
(2.2,4.4) \\
\end{array}$ & $\begin{array}{c}2.7 \\
(1.7,4.4) \\
\end{array}$ & $\begin{array}{c}4.4 \\
(3.0,4.4) \\
\end{array}$ & $\begin{array}{c}4.3 \\
(2.9,4.4) \\
\end{array}$ & $\begin{array}{c}4.2 \\
(1.8,4.4) \\
\end{array}$ & $\begin{array}{c}4.4 \\
(2.3,4.4) \\
\end{array}$ & $p>0.05$ & $p>0.05$ & $p>0.05$ \\
\hline IL-6 & $\begin{array}{c}2.4 \\
(2.4,2.7) \\
\end{array}$ & $\begin{array}{c}2.4 \\
(2.5,3.2) \\
\end{array}$ & $\begin{array}{c}5.3 \\
(2.5,9.8) \\
\end{array}$ & $\begin{array}{c}4.6 \\
(2.7,8.6) \\
\end{array}$ & $\begin{array}{c}3.7 \\
(2.5,7.0) \\
\end{array}$ & $\begin{array}{c}2.9 \\
(2.5,5.9) \\
\end{array}$ & $p>0.05$ & $\begin{array}{l}0.012 \text { (birth-12mo) } \\
0.009 \text { (birth-6-12y) }\end{array}$ & 0.008(birth-12mo) \\
\hline IL-7 & $\begin{array}{c}2.4 \\
(1.9,3.1) \\
\end{array}$ & $\begin{array}{c}2.4 \\
(1.8,2.9) \\
\end{array}$ & $\begin{array}{c}3.1 \\
(2.6,3.6) \\
\end{array}$ & $\begin{array}{c}3.2 \\
(2.1,3.7) \\
\end{array}$ & $\begin{array}{c}2.0 \\
(1.7,3.2) \\
\end{array}$ & $\begin{array}{c}2.1 \\
(1.6,2.9) \\
\end{array}$ & $p>0.05$ & 0.031 (birth-12mo) & $0.023(12 \mathrm{mo}-6-12 \mathrm{y})$ \\
\hline IL-8 & $\begin{array}{c}3.8 \\
(3.8,20.2) \\
\end{array}$ & $\begin{array}{c}5.5 \\
(3.8,7.4) \\
\end{array}$ & $\begin{array}{c}3.8 \\
(3.6,5.7) \\
\end{array}$ & $\begin{array}{c}3.8 \\
(3.4,3.8) \\
\end{array}$ & $\begin{array}{c}3.8 \\
(3.8,3.8) \\
\end{array}$ & $\begin{array}{c}3.8 \\
(3.0,3.8) \\
\end{array}$ & $p>0.05$ & 0.029 (birth-6-12y) & $\begin{array}{l}0.007 \text { (birth-12mo) } \\
0.004 \text { (birth-6-12y) }\end{array}$ \\
\hline IL-10 & $\begin{array}{c}1.4 \\
(1.0,1.9) \\
\end{array}$ & $\begin{array}{c}1.4 \\
(0.9,2.0) \\
\end{array}$ & $\begin{array}{c}4.7 \\
(2.7,10.6) \\
\end{array}$ & $\begin{array}{c}4.1 \\
(1.5,7.4) \\
\end{array}$ & $\begin{array}{c}3.8 \\
(1.4,6.6) \\
\end{array}$ & $\begin{array}{c}2.6 \\
(1.4,14.1) \\
\end{array}$ & $p>0.05$ & $\begin{array}{l}0.001 \text { (birth-12mo) } \\
0.007 \text { (birth-6-12y) }\end{array}$ & $\begin{array}{l}0.001 \text { (birth-12mo) } \\
0.002 \text { (birth-6-12y) }\end{array}$ \\
\hline IL-12 (p70) & $\begin{array}{c}1.1 \\
(0.9,2.1) \\
\end{array}$ & $\begin{array}{c}1.1 \\
(0.8,1.4) \\
\end{array}$ & $\begin{array}{c}3.0 \\
(2.1,5.0) \\
\end{array}$ & $\begin{array}{c}1.9 \\
(1.3,3.7) \\
\end{array}$ & $\begin{array}{c}2.2 \\
(1.3,3.3) \\
\end{array}$ & $\begin{array}{c}1.8 \\
(1.3,3.7) \\
\end{array}$ & $p>0.05$ & $\begin{array}{l}0.001 \text { (birth-12mo) } \\
0.028 \text { (birth-6-12y) }\end{array}$ & $\begin{array}{l}0.001 \text { (birth-12mo) } \\
0.003 \text { (birth-6-12y) }\end{array}$ \\
\hline IL-13 & $\begin{array}{c}2.6 \\
(2.2,2.8) \\
\end{array}$ & $\begin{array}{c}2.4 \\
(2.0,2.6) \\
\end{array}$ & $\begin{array}{c}4.0 \\
(2.9,6.1) \\
\end{array}$ & $\begin{array}{c}4.0 \\
(2.7,5.0) \\
\end{array}$ & $\begin{array}{c}3.7 \\
(2.5,5.5) \\
\end{array}$ & $\begin{array}{c}4.8 \\
(3.1,6.6) \\
\end{array}$ & $p>0.05$ & $\begin{array}{l}0.001 \text { (birth-12mo) } \\
0.019 \text { (birth-6-12y) }\end{array}$ & $\begin{array}{l}0.001 \text { (birth-12mo) } \\
0.001 \text { (birth-6-12y) }\end{array}$ \\
\hline IL-17 & $\begin{array}{c}3.3 \\
(3.0,5.2)\end{array}$ & $\begin{array}{c}3.0 \\
(2.4,4.2)\end{array}$ & $\begin{array}{c}4.1 \\
(3.1,6.0)\end{array}$ & $\begin{array}{c}4.5 \\
(2.9,6.9)\end{array}$ & $\begin{array}{c}3.0 \\
(1.6,4.0)\end{array}$ & $\begin{array}{c}3.0 \\
(2.2,3.2)\end{array}$ & $p>0.05$ & 0.018(birth-12mo) & $0.006(12 \mathrm{mo}-6-12 \mathrm{y})$ \\
\hline IFN- $\gamma$ & $\begin{array}{c}35.5 \\
(25.5,59.7) \\
\end{array}$ & $\begin{array}{c}36.2 \\
(18.7,57.7) \\
\end{array}$ & $\begin{array}{c}43.7 \\
(37.1,62.0) \\
\end{array}$ & $\begin{array}{c}53.2 \\
(30.3,74.0) \\
\end{array}$ & $\begin{array}{c}31.6 \\
(19.4,44.7) \\
\end{array}$ & $\begin{array}{c}31.7 \\
(24.3,42.7) \\
\end{array}$ & $p>0.05$ & 0.029 (12mo-6-12y) & $\begin{array}{c}0.029 \text { (birth-12mo) } \\
0.019 \text { (12mo-6-12y) }\end{array}$ \\
\hline TNF- $\alpha$ & $\begin{array}{c}12.0 \\
(7.2,15.0)\end{array}$ & $\begin{array}{c}8.5 \\
(4.8,11.1) \\
\end{array}$ & $\begin{array}{c}12.8 \\
(10.9,20.7) \\
\end{array}$ & $\begin{array}{c}22.4 \\
(8.7,25.1) \\
\end{array}$ & $\begin{array}{c}7.7 \\
(5.8,13.6) \\
\end{array}$ & $\begin{array}{c}10.5 \\
(6.6,13.4)\end{array}$ & $p>0.05$ & 0.015 (12mo-6-12y) & $\begin{array}{c}0.001 \text { (birth-12mo) } \\
0.031(12 \mathrm{mo}-6-12 \mathrm{y})\end{array}$ \\
\hline G-CSF & $\begin{array}{c}8.9 \\
(5.6,11.4) \\
\end{array}$ & $\begin{array}{c}7.9 \\
(5.4,13.2) \\
\end{array}$ & $\begin{array}{c}11.4 \\
(7.9,16.6) \\
\end{array}$ & $\begin{array}{c}11.3 \\
(6.6,14.7) \\
\end{array}$ & $\begin{array}{c}5.8 \\
(4.2,8.2) \\
\end{array}$ & $\begin{array}{c}6.0 \\
(4.4,8.2) \\
\end{array}$ & $p>0.05$ & $\begin{array}{c}0.049 \text { (birth-6-12y) } \\
0.001 \text { (12mo-6-12y) }\end{array}$ & 0.001 (12mo-6-12y) \\
\hline GM-CSF & $\begin{array}{c}41.3 \\
(25.1,53.9) \\
\end{array}$ & $\begin{array}{c}33.1 \\
(27.9,51.1) \\
\end{array}$ & $\begin{array}{c}43.6 \\
(32.3,52.4)\end{array}$ & $\begin{array}{c}51.3 \\
(25.5,77.9) \\
\end{array}$ & $\begin{array}{c}20.6 \\
(16.5,34.1) \\
\end{array}$ & $\begin{array}{c}26.6 \\
(19.7,34.3) \\
\end{array}$ & $p>0.05$ & $\begin{array}{c}0.004 \text { (birth-6-12y) } \\
0.002 \text { (12mo-6-12y) }\end{array}$ & $\begin{array}{c}0.050 \text { (birth-6-12y) } \\
0.001 \text { (12mo-6-12y) }\end{array}$ \\
\hline MCP-1 & $\begin{array}{c}13.2 \\
(9.2,15.5) \\
\end{array}$ & $\begin{array}{c}14.4 \\
(8.5,26.1) \\
\end{array}$ & $\begin{array}{c}24.0 \\
(19.6,36.0) \\
\end{array}$ & $\begin{array}{c}22.4 \\
(18.5,32.7) \\
\end{array}$ & $\begin{array}{c}17.7 \\
(11.6,45.3) \\
\end{array}$ & $\begin{array}{c}20.1 \\
(15.7,27.6) \\
\end{array}$ & $p>0.05$ & $\begin{array}{l}0.001 \text { (birth-12mo) } \\
0.017 \text { (birth-6-12y) }\end{array}$ & 0.006(birth-12mo) \\
\hline MIP-1 $1 \beta$ & $\begin{array}{c}31.7 \\
(22.5,81.2)\end{array}$ & $\begin{array}{c}41.2 \\
(24.6,93.2)\end{array}$ & $\begin{array}{c}46.0 \\
(29.1,71.0)\end{array}$ & $\begin{array}{c}23.9 \\
(21.5,35.4)\end{array}$ & $\begin{array}{c}28.0 \\
(19.8,61.7)\end{array}$ & $\begin{array}{c}25.2 \\
(19.6,38.8)\end{array}$ & $\begin{array}{c}0.009 \\
(12 \mathrm{mo})\end{array}$ & $p>0.05$ & $\begin{array}{l}0.023 \text { (birth-12mo) } \\
0.028 \text { (birth-6-12y) }\end{array}$ \\
\hline
\end{tabular}

Table 3 - Median T lymphocytes subset percentages in -control and HEU groups at different ages, with interquartile values in parenthesis

\begin{tabular}{|c|c|c|c|c|c|c|c|c|c|}
\hline \multirow[b]{2}{*}{ Parameters } & \multicolumn{2}{|c|}{ Birth } & \multicolumn{2}{|c|}{$12 \mathrm{mo}$} & \multicolumn{2}{|c|}{$6-12 y$} & \multicolumn{3}{|c|}{$p$ values } \\
\hline & Control & HEU & Control & HEU & Control & HEU & $\begin{array}{l}\text { HEU vs. } \\
\text { Control }\end{array}$ & $\begin{array}{c}\text { Control at different } \\
\text { ages }\end{array}$ & $\begin{array}{l}\text { HEU at different } \\
\text { ages }\end{array}$ \\
\hline \multicolumn{10}{|l|}{ CD4+ T cells } \\
\hline \%CD38+ HLA-DR+ & $\begin{array}{c}1.4 \\
(1.2,2.0) \\
\end{array}$ & $\begin{array}{c}1.8 \\
(1.7,2.2) \\
\end{array}$ & $\begin{array}{c}4.0 \\
(2.8,5.9) \\
\end{array}$ & $\begin{array}{c}2.3 \\
(1.5,4.6) \\
\end{array}$ & $\begin{array}{c}1.7 \\
(1.6,2.2) \\
\end{array}$ & $\begin{array}{c}2.9 \\
(2.0,4.9) \\
\end{array}$ & $\begin{array}{l}0.006 \\
(6-12 y)\end{array}$ & $\begin{array}{c}0.005 \text { (birth-12mo) } \\
0.005 \text { (12mo-6-12y) }\end{array}$ & 0.038 (birth-6-12y) \\
\hline$\%$ PD-1+ & $\begin{array}{c}3.2 \\
(2.2,4.5)\end{array}$ & $\begin{array}{c}1.8 \\
(1.3,3.4)\end{array}$ & $\begin{array}{c}10.4 \\
(9.2,14.8)\end{array}$ & $\begin{array}{c}10.9 \\
(5.5,23.5)\end{array}$ & $\begin{array}{c}10.4 \\
(7.4,13.6)\end{array}$ & $\begin{array}{c}12.0 \\
(8.3,18.5)\end{array}$ & $p>0.05$ & $\begin{array}{l}0.008 \text { (birth-12mo) } \\
0.004 \text { (birth-6-12y) }\end{array}$ & $\begin{array}{l}0.002 \text { (birth-12mo) } \\
0.001 \text { (birth-6-12y) }\end{array}$ \\
\hline$\%$ 2B4+ & $\begin{array}{c}1.9 \\
(0.2,2.6)\end{array}$ & $\begin{array}{c}1.7 \\
(0.7,3.1)\end{array}$ & $\begin{array}{c}5.9 \\
(4.2,7.5)\end{array}$ & $\begin{array}{c}2.1 \\
(0.7,5.3)\end{array}$ & $\begin{array}{c}5.4 \\
(2.3,10.0)\end{array}$ & $\begin{array}{c}10.3 \\
(4.4,16.1)\end{array}$ & $p>0.05$ & $\begin{array}{l}0.030 \text { (birth-12mo) } \\
0.004 \text { (birth-6-12y) }\end{array}$ & $\begin{array}{c}0.001 \text { (birth-6-12y) } \\
0.002(12 \mathrm{mo}-6-12 \mathrm{y})\end{array}$ \\
\hline \multicolumn{10}{|l|}{ CD8+ $T$ cells } \\
\hline \%CD38+ HLA-DR+ & $\begin{array}{c}12.1 \\
(9.3,15.9)\end{array}$ & $\begin{array}{c}9.6 \\
(6.8,15.5)\end{array}$ & $\begin{array}{c}12.2 \\
(8.2,15.3)\end{array}$ & $\begin{array}{c}18.6 \\
(16.0,25.6)\end{array}$ & $\begin{array}{c}5.6 \\
(4.4,6.9)\end{array}$ & $\begin{array}{c}7.8 \\
(5.9,14.3)\end{array}$ & $\begin{array}{c}0.053 \\
(12 \mathrm{mo})\end{array}$ & 0.001 (birth-6-12y) & $\begin{array}{c}0.011 \text { (birth-12mo) } \\
0.001(12 \mathrm{mo}-6-12 \mathrm{y})\end{array}$ \\
\hline$\%$ PD-1+ & $\begin{array}{c}7.8 \\
(6.2,11.3)\end{array}$ & $\begin{array}{c}4.9 \\
(4.2,8.0)\end{array}$ & $\begin{array}{c}18.6 \\
(11.5,26.2)\end{array}$ & $\begin{array}{c}15.3 \\
(10.8,24.3)\end{array}$ & $\begin{array}{c}11.6 \\
(7.4,15.1)\end{array}$ & $\begin{array}{c}6.3 \\
(3.7,22.5)\end{array}$ & $p>0.05$ & 0.026(birth-12mo) & 0.009 (birth-12mo) \\
\hline$\% 2 \mathrm{~B} 4+$ & $\begin{array}{c}14.7 \\
(11.9,23.1)\end{array}$ & $\begin{array}{c}14.7 \\
(12.4,20.1)\end{array}$ & $\begin{array}{c}57.4 \\
(41.3,60.4)\end{array}$ & $\begin{array}{c}40.5 \\
(30.9,53.2)\end{array}$ & $\begin{array}{c}44.8 \\
(38.3,56.1)\end{array}$ & $\begin{array}{c}48.9 \\
(44.0,66.0)\end{array}$ & $p>0.05$ & $\begin{array}{l}0.001 \text { (birth-12mo) } \\
0.001 \text { (birth-6-12y) }\end{array}$ & $\begin{array}{l}0.008 \text { (birth-12mo) } \\
0.001 \text { (birth-6-12y) }\end{array}$ \\
\hline
\end{tabular}


The percentage of transitional B cells decreased after 12 months of age in both groups (Table 4). Naïve B cells did not show any statistical difference when groups or ages strata were assessed (Table 4). Resting memory B cell percentages increased from birth and reached the highest levels at 6-12 years for control and HEU groups (Table 4). The percentage of activated mature B cells increased after 12 months in both groups (Table 4). Exhausted memory B cells decreased from birth to 12 months and had a small increase at 6-12 years of age in both groups (Table 4). CD38 expression on B cells decreased after birth in both groups (Table 4).

There were no statistical differences between groups for PD- 1 and FcRL4 expression on B cells ( $\mathrm{p}=0.178, \mathrm{p}=0.066$, respectively), neither was there for PD-1 expression on NK cells $(\mathrm{p}=0.295)$ (Table 4).

Correlation between CD4+ T cell counts and exhaustion and activation markers

A strong inverse correlation between CD4+T cell counts and CD38+HLA-DR+ on CD8+T cells was observed in HEU individuals aged 12 months $(r=-0.677, p=0.032)$.

Similarly, a strong inverse correlation between CD4+T cell counts and PD- 1 expression on CD4+T and CD8+T cells was observed in HEU individuals aged 12 months
$(\mathrm{CD} 4+\mathrm{PD}-1+: \mathrm{r}=-0.655, \mathrm{p}=0.040 ; \mathrm{CD} 8+\mathrm{PD}-1+$ : $\mathrm{r}=-0.730, \mathrm{p}=0.016$ ).

\section{TRECs in PBMC}

The median number of TREC copies/ $10^{6} \mathrm{PBMC}$ on cord samples was similar between HEU and control groups (HEU: 52646, Controls: 64322, $\mathrm{p}=0.800$ ).

\section{DISCUSSION}

The results from this study suggest that HIV-exposed uninfected children have a similar pattern and share many immunological aspects after birth with healthy age-matched controls. Both had low levels of microbial translocation markers in plasma in all age strata with ascending levels from birth to 12 months and a subsequent drop at 6-12 years for sCD14 and concurrently for 12 of the 17 cytokines analyzed. Also, no evidence of damage to thymus function at birth was noted in HEU. There were only a few differences. Among plasma cytokines, HEU had lower plasma MIP- $1 \beta$ levels at 12 months and higher IL-4 levels at 6-12 years when compared to controls at similar age strata. HEU also showed higher activation markers on CD8+ T cells at 12 months and on CD4+ T cells at 6-12 years than the -control group. These differences could be attributed to

Table 4 - Median B and NK cell subsets in control and HEU groups at different ages, with interquartile values in parenthesis

\begin{tabular}{|c|c|c|c|c|c|c|c|c|c|}
\hline \multirow[b]{2}{*}{ Parameters } & \multicolumn{2}{|c|}{ Birth } & \multicolumn{2}{|c|}{$12 \mathrm{mo}$} & \multicolumn{2}{|c|}{ 6-12 y } & \multicolumn{3}{|c|}{$p$ values } \\
\hline & Control & HEU & Control & HEU & Control & HEU & $\begin{array}{l}\text { HEU vs. } \\
\text { Control }\end{array}$ & $\begin{array}{c}\text { Control at different } \\
\text { ages }\end{array}$ & $\begin{array}{c}\text { HEU at different } \\
\text { ages }\end{array}$ \\
\hline \multicolumn{10}{|l|}{ B cells } \\
\hline \%Transitional (CD10+) & $\begin{array}{c}63.1 \\
(58.3,66.7)\end{array}$ & $\begin{array}{c}62.7 \\
(57.6,64.4)(\end{array}$ & $\begin{array}{c}59.1 \\
(56.2,77.0)\end{array}$ & $\begin{array}{c}59.2 \\
(53.0,62.0)\end{array}$ & $\begin{array}{c}49.9 \\
(47.1,50.8)\end{array}$ & $\begin{array}{c}53.9 \\
(52.8,57.0)\end{array}$ & $p>0.050$ & $\begin{array}{c}0.001 \text { (birth-6-12y) } \\
0.001(12 \mathrm{mo}-6-12 \mathrm{y})\end{array}$ & 0.043(birth-6-12y) \\
\hline $\begin{array}{l}\text { \%Naïve } \\
\text { (CD10-CD21+CD27-) }\end{array}$ & $\begin{array}{c}73.7 \\
(58.8,84.8)\end{array}$ & $\begin{array}{c}53.5 \\
(38.2,73.6)(\end{array}$ & $\begin{array}{c}77.5 \\
(69.0,85.5)\end{array}$ & $\begin{array}{c}81.9 \\
(77.1,87.5)\end{array}$ & $\begin{array}{c}75.0 \\
(63.3,78.8)\end{array}$ & $\begin{array}{c}63.3 \\
(53.6,71.6)\end{array}$ & $p>0.050$ & $p>0.050$ & $p>0.050$ \\
\hline $\begin{array}{l}\text { \%Resting memory } \\
\text { (CD10-CD21+CD27+) }\end{array}$ & $\begin{array}{c}0.9 \\
(0.5,1.4) \\
\end{array}$ & $\begin{array}{c}0.8 \\
(0.4,1.6) \\
\end{array}$ & $\begin{array}{c}3.8 \\
(2.0,7.1) \\
\end{array}$ & $\begin{array}{c}2.2 \\
(1.7,4.1) \\
\end{array}$ & $\begin{array}{c}8.5 \\
(6.1,11.6) \\
\end{array}$ & $\begin{array}{c}6.4 \\
(4.7,11.6) \\
\end{array}$ & $p>0.050$ & $\begin{array}{l}0.010 \text { (birth-12mo) } \\
0.001 \text { (birth-6-12y) }\end{array}$ & $\begin{array}{l}0.038 \text { (birth-12mo) } \\
0.001 \text { (birth-6-12y) }\end{array}$ \\
\hline $\begin{array}{l}\text { \% Activated Mature } \\
\text { (CD10-CD21-CD27+) }\end{array}$ & $\begin{array}{c}1.4 \\
(0.8,2.0) \\
\end{array}$ & $\begin{array}{c}2.6 \\
(0.6,3.5) \\
\end{array}$ & $\begin{array}{c}2.8 \\
(1.9,5.1) \\
\end{array}$ & $\begin{array}{c}2.7 \\
(1.8,3.2)\end{array}$ & $\begin{array}{c}4.4 \\
(3.2,8.8)\end{array}$ & $\begin{array}{c}6.1 \\
(4.5,9.7) \\
\end{array}$ & $p>0.050$ & $\begin{array}{l}0.037 \text { (birth-12mo) } \\
0.001 \text { (birth-6-12y) }\end{array}$ & 0.001 (birth-6-12y) \\
\hline $\begin{array}{l}\text { \% Exhausted Memory } \\
\text { (CD10-CD21-CD27-) }\end{array}$ & $\begin{array}{c}23.1 \\
(13.1,39.3)\end{array}$ & $\begin{array}{c}40.2 \\
(22.1,58.1)\end{array}$ & $\begin{array}{c}10.3 \\
(7.9,17.8)\end{array}$ & $\begin{array}{c}11.1 \\
(4.8,13.9)\end{array}$ & $\begin{array}{c}12.8 \\
(7.9,15.9)\end{array}$ & $\begin{array}{c}22.2 \\
(13.2,23.6)\end{array}$ & $p>0.050$ & $\begin{array}{l}0.047 \text { (birth-12mo) } \\
0.040 \text { (birth-6-12y) }\end{array}$ & $\begin{array}{l}0.001 \text { (birth-12mo) } \\
0.047 \text { (birth-6-12y) }\end{array}$ \\
\hline$\%$ CD38+ & $\begin{array}{c}42.8 \\
(29.0,48.5)( \\
\end{array}$ & $\begin{array}{c}37.8 \\
(29.7,21.3)( \\
\end{array}$ & $\begin{array}{c}33.3 \\
(26.0,40.0) \\
\end{array}$ & $\begin{array}{c}35.9 \\
(32.5,39.5) \\
\end{array}$ & $\begin{array}{c}13.1 \\
(9.3,15.4) \\
\end{array}$ & $\begin{array}{c}13.4 \\
(6.6,31.7) \\
\end{array}$ & $p>0.050$ & $\begin{array}{c}0.001 \text { (birth-6-12y) } \\
0.005(12 \mathrm{mo}-6-12 \mathrm{y})\end{array}$ & $\begin{array}{c}0.024 \text { (birth-6-12y) } \\
0.010(12 \mathrm{mo}-6-12 \mathrm{y})\end{array}$ \\
\hline$\%$ PD-1+ & $\begin{array}{c}0.2 \\
(0.0,0.8) \\
\end{array}$ & $\begin{array}{c}0.7 \\
(0.2,1.6)\end{array}$ & $\begin{array}{c}0.1 \\
(0.1,0.1)\end{array}$ & $\begin{array}{c}0.1 \\
(0.1,0.2)\end{array}$ & $\begin{array}{c}0.4 \\
(0.3,0.5)\end{array}$ & $\begin{array}{c}0.5 \\
(0.3,1.9)\end{array}$ & $p>0.050$ & $p>0.050$ & $p>0.050$ \\
\hline \% FcRL4+ & $\begin{array}{c}0.5 \\
(0.3,0.8) \\
\end{array}$ & $\begin{array}{c}1.7 \\
(1.0,2.3) \\
\end{array}$ & $\begin{array}{c}1.9 \\
(1.6,2.4) \\
\end{array}$ & $\begin{array}{c}0.8 \\
(0.7,0.9) \\
\end{array}$ & $\begin{array}{c}3.1 \\
(1.1,6.3)\end{array}$ & $\begin{array}{c}1.1 \\
(0.6,1.9) \\
\end{array}$ & $p>0.050$ & $p>0.050$ & $p>0.050$ \\
\hline \multicolumn{10}{|l|}{ NK cells } \\
\hline$\%$ PD-1+ & $\begin{array}{c}0.7 \\
(0.3,1.6)\end{array}$ & $\begin{array}{c}1.6 \\
(0.6,2.8)\end{array}$ & $\begin{array}{c}0.6 \\
(0.5,0.8)\end{array}$ & $\begin{array}{c}0.6 \\
(0.5,0.7)\end{array}$ & $\begin{array}{c}0.4 \\
(0.2,0.8)\end{array}$ & $\begin{array}{c}1.5 \\
(1.4,2.1)\end{array}$ & $p>0.050$ & $p>0.050$ & $p>0.050$ \\
\hline
\end{tabular}


HIV exposure or may be unrelated. The similarities were greater than the differences, indicating that HEU are not significantly immune compromised. Moreover, this study provided valuable insight into the development of the immune system over the first year of life.

The cytokines IL-2, IL-6, IL-7, IL-10, IL-12p70, IL-13, IL-17, IFN- $\gamma$, TNF- $\alpha$, G-CSF, GM-CSF and MCP-1 followed a similar pattern in the two groups. They showed an increase in the first 12 months of age, lower at 6-12 years. That pattern might reflect exposure to both environmental and vaccine antigens during the first year of life, as previously observed ${ }^{16}$. In contrast, two cytokines, plasma MIP-1 $\beta$ and IL-4 levels followed a different pattern in HEU than in controls.

Plasma MIP-1 $\beta$ levels were higher in HEU at birth as compared to levels at 12 months and at 6-12 years, whereas in controls MIP- $1 \beta$ levels increased from birth to 12 months, such that levels at 12 months were higher in controls than in HEU. MIP- $1 \alpha$, MIP- $1 \beta$ and RANTES are $\beta$-chemokines and as natural ligands of CCR5, they are known to interfere with HIV infection. HIV-exposed uninfected adults at increased risk of infection demonstrate higher $\beta$-chemokine levels than non-HIV-exposed adults, suggesting that $\beta$-chemokines are amongst the factors that prevent HIV infection in some individuals ${ }^{17}$. Recently, some elite controllers were shown to express higher levels of plasma MIP-1 $\beta$ levels and this was sufficient to block R5-tropic HIV entry ${ }^{18}$. In this way, the higher MIP-1 $\beta$ levels at birth when compared to 12 months in the HEU group may indicate an appropriate immune response to a limited period of HIV exposure ${ }^{19}$. The higher IL-4 levels at 6-12 years in HEU that contrasted with controls, could be an indicator of a predominant Th2 response in the HEU group, although we could not corroborate this with additional studies or clinical findings.

Immune activation is a feature of many inflammatory conditions including chronic HIV infection and has been attributed to different factors such as microbial translocation and HIV medicated immune activation. Somewhat surprising was the higher activation markers on CD8+ T cells at 12 months and on CD4+ T cells at 6-12 years in HEU compared to the control group. Other studies have shown higher CD38+ expression on CD8+T cells in HEU than HIVunexposed individuals at different ages ${ }^{14,15}$. This difference might represent transitory immune activation due to HIV exposure in the peripartum and, possibly, a consequent late maturation of the immune system. The inverse correlation observed between CD4+ T cell counts and exhausted/ activated CD8+ T cells reinforce the possibility that, at least for some individuals, an inflammatory environment might contribute to lower CD4+ T cell levels.
No statistical differences were noted between HEU and control groups in respect to B and NK cells at any of the three age-strata analyzed. As most HEU children analyzed in this study had mothers with low viral load copies at delivery, the intensity of HIV exposure was presumably insufficient to have influenced B and NK cells significantly.

The first postnatal year of life seems to be a key period for programming the immune system. Recently, attention has been focused on the establishment of the gut microbiota, since it contributes to the maturation of the immune system ${ }^{1,2,20}$. Feeding and other factors such as use of antibiotics may influence the gut microbial and the intestinal epithelial integrity ${ }^{2,21-23}$. In our study, most HEU individuals were formula-fed,as one of the preventive strategies against vertical HIV infection, and SMZ-TMP was given to all HEU infants. Both the formula-feeding and antibiotic therapy cohort may have interfered in the gut/ airway microbiota establishment, thus contributing to an altered immune system profile in some aspects.

Similar to what was previously shown ${ }^{24}$, we did not find differences between HEU and healthy HIV-unexposed population in regard to the number of TREC in CD4+T cells, suggesting that there is no damage in thymus function.

This study had some limitations. We evaluated a small number of individuals who were not from a longitudinal cohort. Also, similar to exposure to antiretrovirals and antibiotics and to the choice of caesarean section, some characteristics such as breastfeeding and maternal smoking differed between groups. However, those are characteristics of the environment of HEU infants and it is very difficult to isolate the influence of each of these variables on the child's immune system.

Despite the small number of individuals in each group, we demonstrated that HEU infants and children whose mothers were on ARV during pregnancy had few alterations in comparison to healthy controls. We have also looked at long-term consequences by studying older children, and found very little immunological abnormalities or clinical consequences in this cohort. These changes may be due to HIV exposure, mainly in the peripartum, and/or altered microbiota development with consequences such as impairment in the maturation of the immune system, possible imbalance of cytokine production and poor immune response against antigens.

Depending on the intensity of exposure to different stimuli, a different clinical profile might be present. A HIVinfected pregnant woman with an undetectable HIV viral load might expose her fetus to a less modified environment that one with a high viral load and a very activated immune system. In this way, medical advances that contribute to an adequate control of HIV infection and their consequences to 
the maternal immune system do also contribute to provide the fetus with a less modified environment. Of note, none of the HEU children evaluated presented any serious disease.

In summary, our study shows that the immune system of HIV-exposed uninfected children is, for the majority of analyzed parameters, comparable to non-HIV-exposed children. Although many cytokines and activation markers appear to be more intensively expressed in the first year of life, probably due to antigenic exposure, the immune system seems to go back to homeostasis after that period. It is unclear whether the few alterations observed among HIV-exposed uninfected children will turn into immune disorders in the future. More long-term studies that monitor those changes observed in this heterogeneous population might be able to answer that question.

\section{CONFLICT OF INTERESTS}

All authors declare that they have no conflict of interests.

\section{ACKNOWLEDGMENTS}

This study was funded by Fundação de Amparo à Pesquisa do Estado de São Paulo (Fapesp), Brazil (grants No 10/09701-3 and 10/09738-4).

\section{REFERENCES}

1. Sommer F, Bäckhed F. The gut microbiota - masters of host development and physiology. Nat Rev Microbiol. 2013;11:22738.

2. Tamburini S, Shen N, Wu HC, Clemente JC. The microbiome in early life: implications for health outcomes. Nat Med. 2016;22:713-22.

3. Gupta A, Bhosale R, Kinikar A, Gupte N, Bharadwaj R, Kagal A, et al. Maternal tuberculosis: a risk factor for mother-to-child transmission of human immunodeficiency virus. J Infect Dis. 2011;203:358-63.

4. Fiore S, Newell ML, Trabattoni D, Thorne C, Gray L, Savasi V, et al. Antiretroviral therapy-associated modulation of Th1 and Th2 immune responses in HIV-infected pregnant women. J Reprod Immunol. 2006;70:143-50.

5. Frank DN, Manigart O, Leroy V, Meda N, Valéa D, Zhang W, et al. Altered vaginal microbiota are associated with perinatal mother-to-child transmission of HIV in African women from Burkina Faso. J Acquir Immune Defic Syndr. 2012;60:299-306.

6. Watts DH. Mother to child transmission of HIV: another complication of bacterial vaginosis? J Acquir Immune Defic Syndr. 2012;60:221-4.

7. Prieto LM, González-Tomé MI, Muñoz E, Fernández-Ibieta M, Soto B, Del Rosal T, et al. Low rates of mother-to-child transmission of HIV-1 and risk factors for infection in Spain: 2000-2007. Pediatr Infect Dis J. 2012;31:1053-8.

8. American Academy of Pediatrics. Human immunodeficiency virus infection. In: Kimberlin DW, editor. Red Book: 2015 report of the Committee on Infectious Diseases. $30^{\text {th }}$ ed. Elk-Grove Village: American Academy of Pediatrics; 2015. p.453-76.

9. Afran L, Garcia Knight M, Nduati E, Urban BC, Heyderman RS, Rowland-Jones SL. HIV-exposed uninfected children: a growing population with a vulnerable immune system? Clin Exp Immunol. 2014;176:11-22.

10. Cutland CL, Schrag SJ, Zell ER, Kuwanda L, Buchmann E, Velaphi SC, et al. Maternal HIV infection and vertical transmission of pathogenic bacteria. Pediatrics. 2012;130:e581-90.

11. Slogrove AL, Cotton MF, Esser MM. Severe infections in HIV-exposed uninfected infants: clinical evidence of immunodeficiency. J Trop Pediatr. 2010;56:75-81.

12. Mussi-Pinhata MM, Motta F, Freimanis-Hance L, de Souza R, Szyld E, Succi RC, et al. Lower respiratory tract infections among human immunodeficiency virus-exposed, uninfected infants. Int J Infect Dis. 2010;14 Suppl 3:e176-82.

13. Miyamoto M, Pessoa SD, Ono E, Machado DM, Salomão R, Succi RC, et al. Low CD4+ T-cell levels and B-cell apoptosis in vertically HIV-exposed noninfected children and adolescents. J Trop Pediatr. 2010;56:427-32.

14. Clerici M, Saresella M, Colombo F, Fossati S, Sala N, Bricalli $\mathrm{D}$, et al. T-lymphocyte maturation abnormalities in uninfected newborns and children with vertical exposure to HIV. Blood. 2000;96:3866-71.

15. Ono E, Nunes dos Santos AM, de Menezes Succi RC, Machado DM, de Angelis DS, Salomão R, et al. Imbalance of naive and memory $\mathrm{T}$ lymphocytes with sustained high cellular activation during the first year of life from uninfected children born to HIV-1-infected mothers on HAART. Braz J Med Biol Res. 2008;41:700-8.

16. Buck RH, Cordle CT, Thomas DJ, Winship TR, Schaller JP, Dugle JE. Longitudinal study of intracellular T cell cytokine production in infants compared to adults. Clin Exp Immunol. 2002;128:490-7.

17. Shieh B, Yan YP, Ko NY, Liau YE, Liu YC, Lin HH, et al. Detection of elevated serum beta chemokine levels in seronegative Chinese individuals exposed to human immunodeficiency virus type 1. Clin Infect Dis. 2001;33:273-9.

18. Walker WE, Kurscheid S, Joshi S, Lopez CA, Goh G, Choi M, et al. Increased levels of macrophage inflammatory proteins result in resistance to R5-tropic HIV-1 in a subset of elite controllers. J Virol. 2015;89:5502-14.

19. Maurer M, von Stebut E. Macrophage inflammatory protein-1. Int J Biochem Cell Biol. 2004;36:1882-6.

20. Renz H, Brandtzaeg P, Hornef M. The impact of perinatal immune development on mucosal homeostasis and chronic inflammation. Nat Rev Immunol. 2011;12:9-23. 
21. Bisgaard H, Li N, Bonnelykke K, Chawes BL, Skov T, PaludanMüller G, et al. Reduced diversity of the intestinal microbiota during infancy is associated with increased risk of allergic disease at school age. J Allergy Clin Immunol. 2011;128:64652.

22. Martin R, Nauta AJ, Ben Amor K, Knippels LM, Knol J, Garssen J. Early life: gut microbiota and immune development in infancy. Benef Microbes. 2010;1:367-82.
23. Penders J, Thijs C, Vink C, Stelma FF, Snijders B, Kummeling I, et al. Factors influencing the composition of the intestinal microbiota in early infancy. Pediatrics. 2006;118:511-21.

24. Kolte L, Rosenfeldt V, Vang L, Jeppesen D, Karlsson I, Ryder LP, et al. Reduced thymic size but no evidence of impaired thymic function in uninfected children born to human immunodeficiency virus-infected mothers. Pediatr Infect Dis J. 2011;30:325-30. 